\section{References}

1. Calina D, Docea AO, Petrakis D, et al. Towards effective COVID-19 vaccines: updates, perspectives and challenges (review). Int J Mol Med 2020;46:3-16.

2. Guidry JPD, Perrin PB, Laestadius LI, et al. US public support for COVID-19 vaccine donation to low- and middle-income countries during the COVID-19 pandemic. Vaccine 2021;39:2452-2457.

3. Liu Y, Salwi S, Drolet BC. Multivalue ethical framework for fair global allocation of a COVID-19 vaccine. J Med Ethics 2020;46:499-501.

4. Callaway E. The unequal scramble for coronavirus vaccines-by the numbers. Nature 2020;584:506-507.
5. Acharya KP, Ghimire TR, Subramanya SH. Access to and equitable distribution of COVID-19 vaccine in low-income countries. NPJ Vaccines 2021;6:54.

6. Unequal vaccine distribution self-defeating: World Health organization chief tells economic and social council's special ministerial meeting. ReliefWeb website. https://reliefweb.int/report/world/unequal-vaccine-distribution-self-defeatingworld-health-organization-chief-tells. Accessed April 20, 2021.

7. Sawal I, Ahmad S, Tariq W, Tahir MJ, Essar MY, Ahmed A. Unequal distribution of COVID-19 vaccine: a looming crisis. J Med Virol 2021. doi: 10 1002/jmv.27031.

8. Unequal vaccine hesitancy. DAWN website. https://www.dawn.com/news/ 1617613. Published April 11, 2021. Accessed June 11, 2021.

\title{
Adverse events and humoral response after two doses of severe acute respiratory coronavirus virus 2 (SARS-CoV-2) mRNA vaccine in the hospital personnel of a cardiopulmonary tertiary-care center
}

\author{
Erica De Vita MD ${ }^{1,2, a}$, Francesco Sbrana MD ${ }^{1, a}$ (i) , Filippo Quattrone $\mathrm{MD}^{2,3}$, Beatrice Dal Pino MD ${ }^{1}$, Monica Megaro MSc ${ }^{1}$, \\ Roberta Lombardi MSc ${ }^{1}$, Concetta Prontera $\mathrm{PhD}^{1}$, Claudio Passino MD, PhD ${ }^{1,3}$ and Maurizio Petrillo MD \\ ${ }^{1}$ Fondazione Toscana “Gabriele Monasterio," Pisa, Italy, ${ }^{2}$ Management and Healthcare Laboratory, Institute of Management and Department, EMbeDS, Scuola \\ Superiore Sant'Anna, Pisa, Italy and ${ }^{3}$ Institute of Life Sciences, Scuola Superiore Sant'Anna, Pisa, Italy
}

To the Editor-Patients with cardiovascular and chronic obstructive pulmonary disease are at increased risk of severe coronavirus disease 2019 (COVID-19). ${ }^{1}$ The hospital environment is particularly prone to severe acute respiratory syndrome coronavirus 2 (SARS-CoV-2) diffusion; therefore, it is imperative to protect patients and healthcare personnel against COVID-19 outbreaks. COVID-19 vaccination of these populations is the pillar of an effective hospital COVID-19 infection and prevention control (IPC) strategy. To increase its acceptancy and ensure its success, monitoring activities should also be conducted a local level to provide feedback to the targeted populations.

Fondazione Toscana Gabriele Monasterio (FTGM) is a public, tertiary-level, cardiological, pneumological, and heart surgery center with 2 sites, in Pisa and Massa (Italy). It has a cardiac catheterization laboratory hub for acute coronary syndrome and an adult and pediatric cardiac surgery center, and it serves as a referral center for heart failure and primitive pulmonary hypertension patients. FTGM has 123 beds and >5,000 hospital admissions per year. At the beginning of January 2021, according to national and regional regulations, our center started a COVID-19 vaccination campaign for healthcare personnel. The first phase included health workers, administrative staff, and support personnel. The second phase started in March 2021 with an additional vaccination campaign for all outpatients registered on the Tuscany regional COVID-19 vaccination web platform. In the first phase, only Pfizer-BioNTech COVID-19 was used. In the second phase, Pfizer-BioNTech and Moderna vaccines were administered according to Italian clinical recommendations in force.

\footnotetext{
Author for correspondence: Francesco Sbrana, E-mail: francesco.sbrana@ftgm.it

${ }^{\mathrm{a}}$ Authors of equal contribution.

Cite this article: De Vita E, et al. (2022). Adverse events and humoral response after two doses of severe acute respiratory coronavirus virus 2 (SARS-CoV-2) mRNA vaccine in the hospital personnel of a cardiopulmonary tertiary-care center. Infection Control \& Hospital Epidemiology, 43: 1532-1534, https://doi.org/10.1017/ice.2021.321
}

Our retrospective analysis covers the period between January and May 2021. We evaluated the reported adverse effects (AEs) and the humoral response of the first phase of the vaccination campaign against COVID-19. During the first 10 weeks of the study period, 589 of 811 employees (85\%) completed the vaccination cycle, receiving 2 vaccine doses of Pfizer-BioNTech COVID-19 vaccine on day 1 and day 21 ; of these, $82 \%$ were healthcare workers.

At the beginning of March, we invited all people working in FTGM to fill in a questionnaire that included demographic data, medical history, COVID-19-related anamnesis, and local and systemic AE reporting.

Furthermore, 1 month after the second vaccine dose, a serological test to anti-SARS-Cov-2 IgG (Abbott, index value $<1.4$ ) and anti-SARS-Cov-2 receptor binding domain (RBD) spike protein antibodies (Abbott, normal range $<50 \mathrm{AU} / \mathrm{mL}$ ) was offered to healthcare workers and administrative staff. We collected data for 272 workers (response rate, 46\%).

Clinical characteristics of our population are summarized in Table 1; 14 employees who filled out the questionnaire claimed mild COVID disease before vaccination.

Adverse events were recorded in $50 \%$ and $67 \%$ of study participants, respectively, after the first and second vaccine doses. Systemic events are more frequent after the second vaccine dose. The postvaccination adverse events (AEs) are summarized in Fig. 1.

Furthermore, participants who reported AEs required antipyretic medication in $20 \%$ of cases after the first dose and $58 \%$ after the second dose. In most cases, adverse effects lasted $<3$ days (grade I according to MedDRA classification ${ }^{2}$ ). After the first dose, we recorded 3 severe AEs that required hospital monitoring due to systemic symptoms. One of these AEs occurred in a study participant with previous COVID-19 disease who required steroid and antihistamine therapy. The second vaccine dose was administered to 271 of 272 patients; 1 participant preferred not to receive the second dose due to previous COVID-19 disease. 
Table 1. Clinical Characteristics of Study Participants

\begin{tabular}{|c|c|}
\hline Characteristic & Participants $(\mathrm{N}=272)$, No $(\%)$ \\
\hline Age, $y \pm S D$ & $44 \pm 10$ \\
\hline Sex, male & $80(29)$ \\
\hline Body mass index \pm SD & $24 \pm 1.0$ \\
\hline Previous allergies & $84(31)$ \\
\hline Current smoker & $45(16)$ \\
\hline Anxiety & $25(9)$ \\
\hline Arterial hypertension & $20(7)$ \\
\hline Asthma & $16(6)$ \\
\hline Active steroid or chemotherapy & $5(2)$ \\
\hline Diabetes mellitus & $4(1)$ \\
\hline Chronic kidney failure & $1(1)$ \\
\hline Previous COVID-19 & $14(5)$ \\
\hline Within $3 \mathrm{mo}$ & 5 \\
\hline$>6 \mathrm{mo}$ & 9 \\
\hline
\end{tabular}

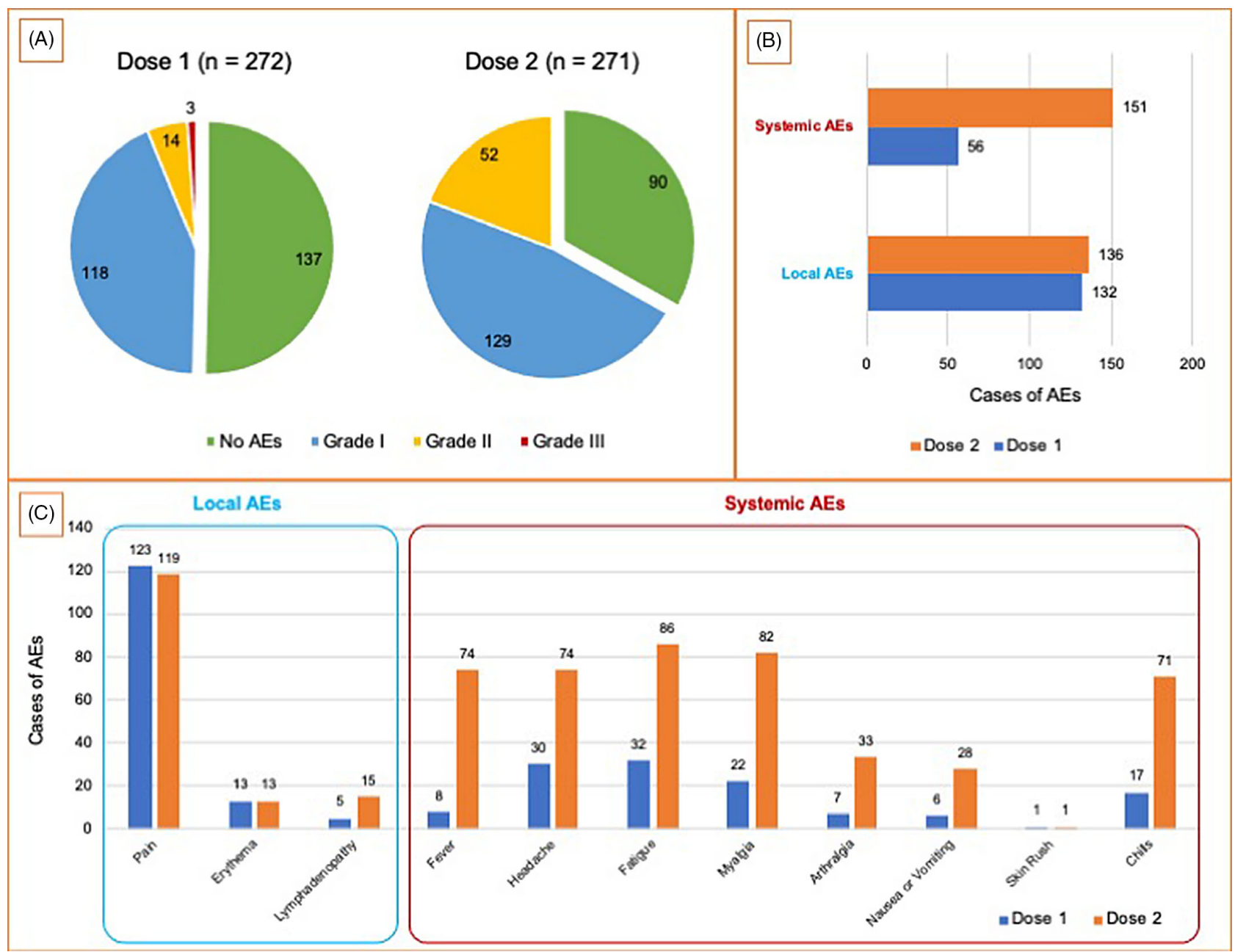

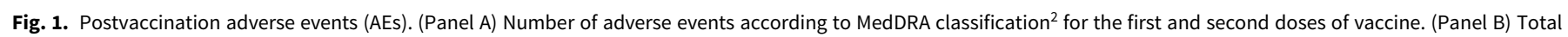
systemic AEs and local AEs for the first and second doses of vaccine. (Panel C) Details of systemic AEs and local AEs for the first and second doses of vaccine. 
Among 272 study participants, 1 had COVID-19 disease within 3 months of follow-up. We found low anti-SARS-Cov-2 IgG values (index value $<1.4$ ) at the serological test in 5 of 14 patients with previous COVID-19 infection. All 5 of these study participants had had COVID-19 disease in the previous 6 months.

A preliminary analysis of the data shows a positive correlation between reporting AEs following the second administration and anti-SARS-Cov-2 RBD spike protein antibodies response. The antibody value in patients with AEs at the second dose was $15,664 \pm 10,640 \mathrm{AU} / \mathrm{mL}$ versus $9,136 \pm 7,523 \mathrm{AU} / \mathrm{mL}$ in patients who did not report adverse effects $(P<.05)$.

In 4 patients, the anti-SARS-Cov- 2 receptor binding domain (RBD) spike protein antibody level was $<600 \mathrm{AU} / \mathrm{mL}$, which indicates very poor humoral immune response to vaccine. Of these, 2 patients were on active chemotherapy, 1 had previous hematological malignancy, and 1 did not report any major issues. These patients were referred to the preventive medicine service for dedicated follow-up. According to some studies, cancer patients could need additional doses of vaccine and/or should maintain nonpharmacological preventive measures as suggested for patients who use B-lymphocyte-depleting agents. ${ }^{3}$

In the first phase of the campaign, $85 \%$ of the staff chose to receive the vaccine. Currently, adherence to the vaccination program is progressively improving to $92 \%$. This result is better than that in a similar Canadian experience in which $80.9 \%$ of the staff accepted the vaccine. ${ }^{4}$ This result is even more significant when we consider that in November 2020, a survey administered to FTGM employees to explore vaccination hesitancy in healthcare and nonhealthcare workers found that just 232 (59\%) of 396 participants had an intention to be vaccinated against COVID-19. We believe that scheduling of vaccinations outside working hours during the weekend, one-to-one vaccine counseling to hesitant workers, peer pressure from colleagues directly involved in the vaccination campaign, and the introduction of a national vaccination mandate for healthcare workers at the end of May played a significant role in achieving the current vaccination rate.

These AE data are not in line with manufacturer-sponsored studies, ${ }^{5}$ but they reflect another single-center experience. ${ }^{6}$ The main limitations of this study are that it is a single-center experience with a short follow-up. We have planned a serological follow-up at 6 and 12 months after the vaccination. Through a dedicated questionnaire, all participants are invited to record any cases of COVID-19 disease to monitor the vaccine's long-term protection. Furthermore, these data will be included in a surveillance network involving all hospitals in Tuscany, which is being used to monitor the evolution of SARS$\mathrm{CoV}-2$ immune response in healthcare workers.

In our opinion, independent studies of real-life acceptancy, safety, and effectiveness of SARS-CoV-2 on high-risk cohorts, such as hospital personnel, are required to boost confidence in vaccine campaigns, both in the general population and in healthcare providers.

Acknowledgments. We thank all the Fondazione Toscana Gabriele Monasterio staff who contributed to the success of the vaccination campaign. In particular, we thank the Hospital Pharmacy Unit, the Laboratory Medicine Unit, the Technical and Rehabilitation Nursing Service (SITRA).

Financial support. No financial support was provided relevant to this article.

Conflicts of interest. All authors report no conflicts of interest relevant to this article.

\section{References}

1. Richardson S, Hirsch JS, Narasimhan M, et al. Presenting characteristics, comorbidities, and outcomes among 5,700 patients hospitalized with COVID-19 in the New York City area. JAMA 2020. doi: 10.1001/jama. 2020.6775 .

2. MedDRA version 23.0 update to be produced. Medical Dictionary for Regulatory Activities website. https://www.meddra.org/news-and-events/ news/meddra-version-230-update-be-produced. Published April 10, 2020. Accessed July 13, 2021.

3. Connolly CM, Boyarsky BJ, Ruddy JA, et al. Absence of humoral response after two-dose SARS-CoV-2 messenger RNA vaccination in patients with rheumatic and musculoskeletal diseases: a case series. Ann Intern Med 2021. doi: 10.7326/M21-1451.

4. Dzieciolowska S, Hamel D, Gadio S, et al. COVID-19 vaccine acceptance, hesitancy, and refusal among Canadian healthcare workers: a multicenter survey. Am J Infect Control 2021. doi: 10.1016/j.ajic.2021.04.079.

5. Polack FP, Thomas SJ, Kitchin N, et al. C4591001 Clinical Trial Group. Safety and efficacy of the BNT162b2 mRNA COVID-19 vaccine. N Engl J Med 2020;383:2603-2615.

6. Riad A, Pokorná A, Attia S, Klugarová J, Koščík M, Klugar M. Prevalence of COVID-19 vaccine side effects among healthcare workers in the Czech Republic. J Clin Med 2021;10:1428.

\title{
Healthcare facilities should publicly report the coronavirus disease 2019 (COVID-19) vaccination coverage of healthcare personnel
}

\author{
Jerry M. Yang ${ }^{1}$, Hilary M. Babcock MD, MPH $^{2}$ and Jonathan D. Baghdadi MD, $\mathrm{PhD}^{3}$ (1) \\ ${ }^{1}$ University of Maryland, College Park, Maryland, ${ }^{2}$ Department of Medicine, Washington University School of Medicine, St. Louis, Missouri and ${ }^{3}$ Department of \\ Epidemiology and Public Health, University of Maryland School of Medicine, Baltimore, Maryland
}

\footnotetext{
edu

Author for correspondence: Jonathan D. Baghdadi, E-mail: jbaghdadi@som.umaryland.

Cite this article: Yang JM, Babcock HM, and Baghdadi JD. (2022). Healthcare facilities should publicly report the coronavirus disease 2019 (COVID-19) vaccination coverage of healthcare personnel. Infection Control \& Hospital Epidemiology, 43: 1534-1536, https:// doi.org/10.1017/ice.2021.319
}

To the Editor-Between expanding eligibility criteria and the opening of mass vaccination sites, millions of Americans have now been vaccinated against coronavirus disease 2019 (COVID19). Barriers to vaccination remain, however, including supply shortages, difficulties in scheduling, and vaccine hesitancy. Among healthcare personnel (HCP), hesitancy has been linked to concerns about adverse effects, a desire to hear the experiences 